\title{
Bleeding from Venous Hemangioma in the Palate Mimicking Hemoptysis
}

\author{
Hyean-Ji Kim, Ha-Yeon Lee, Shin-Hye Yoo, Soo-Im Shin, Jeong-Han Kim, Chang-Hoon Lee \\ Division of Pulmonary and Critical Care Medicine, Department of Internal Medicine, Seoul National University Hospital, Seoul, Korea
}

\begin{abstract}
When physicians meet patients who expectorate blood, hemoptysis should be distinguished from pseudohemoptysis, i.e., bleeding from the upper respiratory tract or upper gastrointestinal tract. Herein, we present a case of hemangioma in the palate that caused bleeding mimicking hemoptysis. Hemangioma in the palate occurs rarely, but it can be easily diagnosed and the patient may avoid unnecessary tests if a physician examines the oral cavity closely at the initial visit.
\end{abstract}

Keywords: Hemoptysis; Hemangioma

\section{INTRODUCTION}

Hemoptysis is the act of coughing up blood or bloodstained mucus from the lower respiratory tract. In general, the evaluation of patients with hemoptysis focuses on exclusion of pulmonary malignancy. However, bleeding from the upper aerodigestive tract (including the oral cavity), such as pseudohemoptysis, can mimic bleeding originating from the lower respiratory tract.

Up to $10 \%$ of patients with initial diagnoses of hemoptysis may be later diagnosed with pseudohemoptysis. Upper aerodigestive tract cancers were identified in $2.1 \%$ of 471 patients with an initial diagnosis of hemoptysis over a six-year period [1]. Thus, when physicians care for patients with blood expectoration, the possibility of upper aerodigestive tract malignancy must be considered. There are various causes of upper aerodigestive tract bleeding. For example, bleeding from vascular anomalies in the oral cavity may be a case of pseudohemoptysis.

We report an uncommon case of hemangioma in the oral cavity, which was initially misdiagnosed as cryptogenic hemoptysis and was finally diagnosed correctly by simple physical examination.

\section{CASE REPORT}

A 39-year-old Korean woman visited emergency room because of recurrent blood expectoration. The symptom developed a year ago and recurred 4 and 8 months later. The first time, she immediately reported to the nearest hospital and underwent evaluation for hemoptysis, including laboratory studies, chest radiograph/ computed tomography scan, flexible bronchoscopy, and angiography. However, all the test results were normal, and the cause of hemoptysis remained unknown. Because blood expectoration recurred, she visited hospital.

She was a previously healthy never-smoker; her past medical and family histories were unremarkable. The patient wasn't taking any medicines, including anticoagulants or antiplatelet drugs. Laboratory studies in the emergency room revealed neither coagulopathy nor thrombocytopenia.

It was observed that she spit blood out of her mouth without coughing or nausea. The expectoration was grossly bloody in the absence of any mucus or food material, the amount less than 2 mL. Blood expectoration was not coincident with menses.
Correspondence to: Chang-Hoon Lee

Division of Pulmonary and Critical Care Medicine, Department of Internal Medicine, Seoul National University Hospital, 101 Daehak-ro, Jongno-gu, Seoul 03080, Korea

Tel: +82-2-2072-4743, Fax: +82-2-762-9662, E-mail: kauri670@empal.com

Received: Apr. 25, 2016 / Accepted after revision: Jun. 1, 2016
(C) 2016 Soonchunhyang Medical Research Institute This is an Open Access article distributed under the terms of the Creative Commons Attribution Non-Commercial License (http://creativecommons.org/licenses/by-nc/4.0/). 


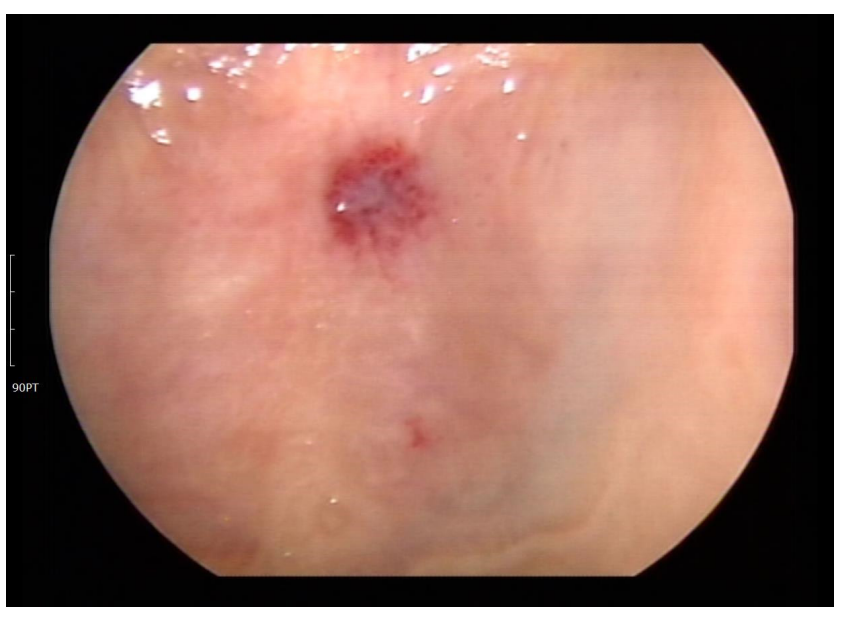

Fig. 1. The laryngoscopic finding of hemangioma in the palate.

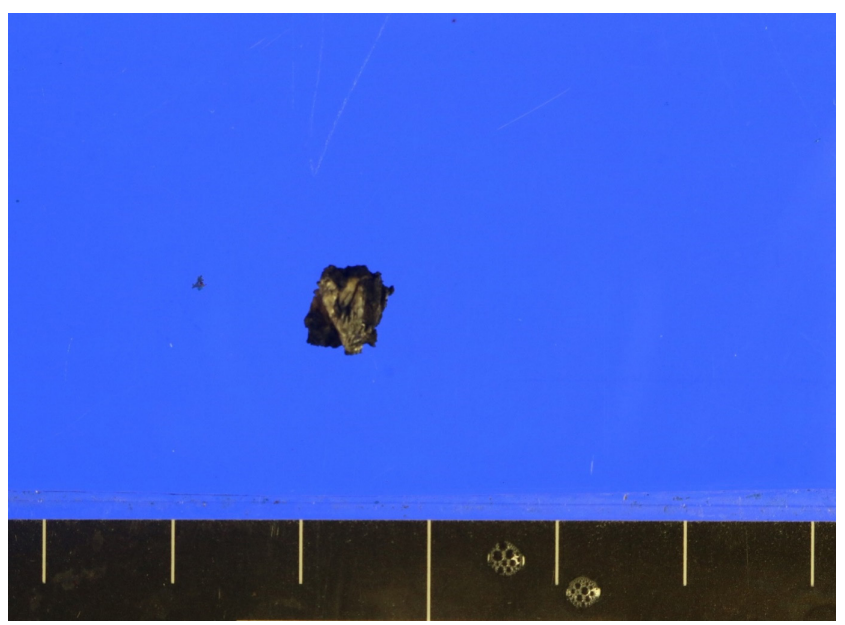

Fig. 2. The gross finding of surgically removed hemangioma.

She did not report cough, increased sputa, nasal stuffiness, runny nose, febrile sensation, rigor, globus symptom, regurgitation, nausea, epigastric soreness, indigestion, hematemesis, melena, or weight loss. Oral cavity exam revealed an approximately 7-mmsized, non-tender, dark red macula on the hard palate. The lesion bled when the patient pressed her tongue against it and suck it. Bleeding was not profuse and stopped with application of mild compression. The patient had not noticed the lesion before (Fig. 1). Since clinical evaluation revealed neither evidences of bleeding tendency nor true hemoptysis, the tentative diagnosis was that bleeding of the lesion in the oral cavity mimicked hemoptysis.

She was referred to an otorhinolaryngologist for diagnostic and therapeutic excision of the lesion. Histopathology examination of surgically excised tissue (Fig. 2) revealed irregularly dilated vascular channels consistent with venous hemangioma (Fig. 3).

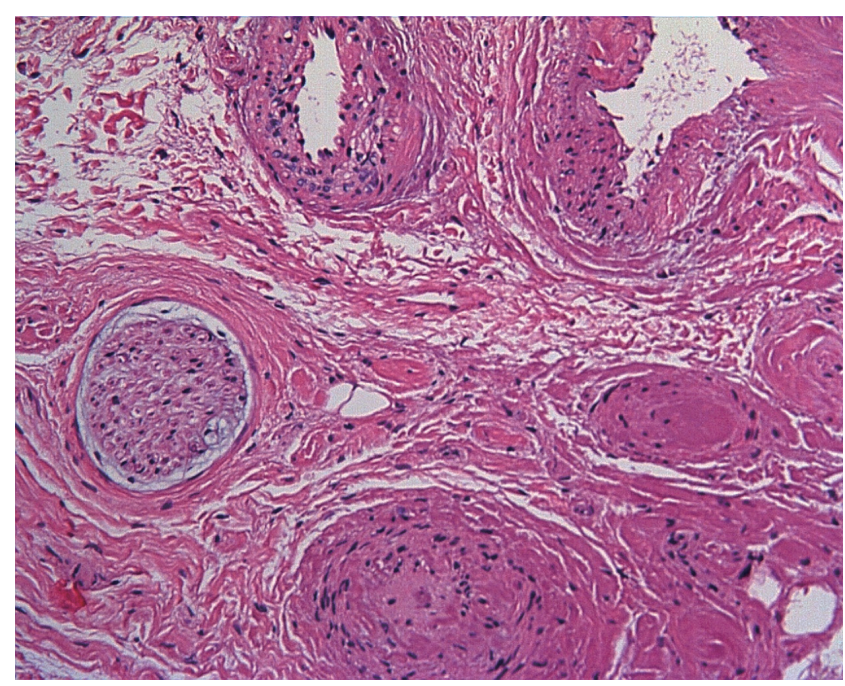

Fig. 3. The histopathology of hemangioma $\left(H \& E_{1} \times 200\right)$.

Two weeks after surgery, the patient revisited outpatient department. The affected area had completely healed without any complications, and she was scheduled to receive regular monthly followup. Thus far, there have been no signs of recurrent 'hemoptysis.'

\section{DISCUSSION}

Non-massive hemoptysis (less than $200 \mathrm{~mL}$ per day) results from multiple causes. Although the most common etiology is bronchitis [2], significant pulmonary diseases such as bronchiectasis/cystic fibrosis, tuberculosis, and bronchogenic carcinoma can cause hemoptysis [3]. When physicians meet patients who expectorate blood, they should pay attention in order to avoid missing these diseases.

The initial examination for hemoptysis is chest radiography. In case of negative findings on chest radiography, it is advised to perform multi-detector computed tomography (MDCT) [4].

In high-risk patients with lung cancer, bronchoscopy could be considered before MDCT. If MDCT shows negative results but hemoptysis resolves, physicians could consider ceasing further investigation, whereas bronchoscopy is warranted if the hemoptysis persists [5].

In cases of recurrent bleeding, physicians could perform angiography [6]. If a cause cannot be identified after bronchoscopy and the patient is not receiving any anticoagulant therapy, cryptogenic hemoptysis should be considered. Up to $42 \%$ of hemoptysis cases are categorized as cryptogenic [7].

However, even after hemoptysis has been considered as crypto- 
genic, it can be later diagnosed as pseudohemoptysis, as in the present case. A guideline states that the first step of evaluating a case of non-massive blood expectoration is to distinguish hemoptysis from pseudohemoptysis. In order to do so, thorough history taking and physical examination are invaluable. According to a previous report, $30 \%$ of patients with hemoptysis report the site of bleeding before inquiries. Fifty percent of patients predict the origin of bleeding with great accuracy, whereas $43 \%$ of physicians identify the origin by physical examination [8].

The present patient underwent various evaluations for hemoptysis, including an angiogram, no cause was found. Almost 1 year later, she was promptly diagnosed with hemangioma by simple physical examination alone.

Oronasal bleeding and hematemesis are common causes of pseudohemoptysis. In general, oronasal bleeding is traumatic [9], and the bleeding focus could be identified by physical examination of the oral cavity and by anterior rhinoscopy of the oropharynx and nasopharynx.

Hematemesis originates from the upper gastrointestinal tract and is usually accompanied by nausea, vomiting, abdominal pain, and previous melena. Blood or coffee ground-like material can be detected during nasogastric lavage.

We presented a case with bleeding hemangioma in the hard palate, one possible cause of oronasal bleeding. There are 2 categories of vascular anomalies in the head and neck: vascular neoplasm (formerly described as hemangioma) and vascular malformation. Arteriovenous malformation of the upper airway is a rare vascular anomaly, but when present, it is persistent and progressive and can represent a lethal benign disease [10].

Hemangioma and vascular malformation have similar pathology. The presence of mitoses on histopathology examination, which is evidence of increased endothelial cell turnover, is the key factor in distinguishing hemangioma from vascular malformation. Although this case of hemangioma in the palate was a rare one, it could have been easily diagnosed and the patient might not have undergone unnecessary testing if a physician had examined her oral cavity closely at the initial visit.

In conclusion, we report a case of bleeding due to hemangioma in the palate, which was initially misdiagnosed as hemoptysis. A meticulous physical examination should be emphasized in the clinical approach for patients who present with blood expectoration.

\section{REFERENCES}

1. DiLeo MD, Amedee RG, Butcher RB. Hemoptysis and pseudohemoptysis: the patient expectorating blood. Ear Nose Throat J 1995;74:822-4, 826,828 .

2. Johnston H, Reisz G. Changing spectrum of hemoptysis: underlying causes in 148 patients undergoing diagnostic flexible fiberoptic bronchoscopy. Arch Intern Med 1989;149:1666-8.

3. Dave BR, Sharma A, Kalva SP, Wicky S. Nine-year single-center experience with transcatheter arterial embolization for hemoptysis: mediumterm outcomes. Vasc Endovascular Surg 2011;45:258-68.

4. Knott-Craig CJ, Oostuizen JG, Rossouw G, Joubert JR, Barnard PM. Management and prognosis of massive hemoptysis: recent experience with 120 patients. J Thorac Cardiovasc Surg 1993;105:394-7.

5. O’Neil KM, Lazarus AA. Hemoptysis: indications for bronchoscopy. Arch Intern Med 1991;151:171-4

6. Samara KD, Tsetis D, Antoniou KM, Protopapadakis C, Maltezakis G, Siafakas NM. Bronchial artery embolization for management of massive cryptogenic hemoptysis: a case series. J Med Case Rep 2011;5:58.

7. Boulay F, Berthier F, Sisteron O, Gendreike Y, Blaive B. Seasonal variation in cryptogenic and noncryptogenic hemoptysis hospitalizations in France. Chest 2000;118:440-4.

8. Pursel SE, Lindskog GE. Hemoptysis: a clinical evaluation of 105 patients examined consecutively on a thoracic surgical service. Am Rev Respir Dis 1961;84:329-36.

9. Schlosser RJ. Clinical practice: epistaxis. N Engl J Med 2009;360:784-9.

10. Manjunath SM, Shetty S, Moon NJ, Sharma B, Metta KK, Gupta N, et al. Arteriovenous malformation of the oral cavity. Case Rep Dent 2014; 2014:353580. 